\title{
Prediction of Locomotor Activity by Infrared Motion Detector on Sleep-wake State in Mice
}

\author{
Jeonghyun Park', Min Soo Jung², Eunsoo Moon ${ }^{1,3}$, Hyun Ju Lim¹, Chi Eun $\mathrm{Oh}^{4}$, Jung Hyun Lee \\ ${ }^{1}$ Department of Psychiatry and Biomedical Research Institute, Pusan National University Hospital, Busan, ${ }^{2}$ Maumpyeonhan Psychiatric Clinic, \\ Changwon, ${ }^{3}$ Department of Psychiatry, Pusan National University School of Medicine, Yangsan, ${ }^{4}$ Department of Pediatrics, Kosin University \\ College of Medicine, Busan, Korea
}

\begin{abstract}
Objective: Behavioral assessments that effectively predict sleep-wake states were tried in animal research. This study aimed to examine the prediction power of an infrared locomotion detector on the sleep-wake states in ICR (Institute Cancer Research) mice. We also explored the influence of the durations and ways of data processing on the prediction power.

Methods: The locomotor activities of seven male mice in home cages were recorded by infrared detectors. Their sleep-wake states were assessed by video analysis. Using the receiver operating characteristic curve analysis, the cut-off score was determined, then the area under the curve $(A \cup C)$ values of the infrared motion detector that predicted sleep-wake states were calculated. In order to improve the prediction power, the four ways of data processing on the prediction power were performed by Matlab 2013b.

Results: In the initial analysis of raw data, the AUC value was 0.785 , but it gradually reached to 0.942 after data summation. The simple data averaging and summation among four different methods showed the maximal AUC value. The 10-minute data summation improved sensitivity (0.889) and specificity $(0.901)$ significantly from the baseline value (sensitivity 0.615; specificity 0.936) ( $p<0.001)$.

Conclusion: This study suggests that the locomotor activity measured by an infrared motion detector might be useful to predict the sleep-wake states in ICR mice. It also revealed that only simple data summation may improve the predictive power. Using daily locomotor activities measured by an infrared motion detector is expected to facilitate animal research related to sleep-wake states.
\end{abstract}

KEY WORDS: Infrared detector; Locomotion; Sleep-wake state; Circadian rhythm; Validity; Mlog system.

\section{INTRODUCTION}

Animal studies are effective to explore the pathophysiology of mental disorders and to initially evaluate the efficacy of drugs that are difficult to perform in human studies [1-6]. Even though some animal studies have faced with difficulties to find proper animal models, animal research on sleep-wake cycles related to sleep disorders or circadian rhythm disorders could be beneficial due to the com-

Received: June 18, 2020 / Revised: July 26, 2020

Accepted: August 4, 2020

Address for correspondence: Eunsoo Moon

Department of Psychiatry and Biomedical Research Institute, Pusan National University Hospital, Pusan National University School of Medicine, 179 Gudeok-ro, Seo-gu, Busan 49241, Korea E-mail: esmun@hanmail.net

ORCID: https://orcid.org/0000-0002-8863-3413 monality of sleep-wake states between human and animal [7]. Sleep-wake states can be precisely measured by electroencephalography (EEG) and electromyography (EMG) on the basis of rapid eye movement (REM) and non-REM [8-11].

However, there are several problems with employing EEG in research. EEG leads could be broken when the animal moves actively that the EEG electrodes are usually placed on the brain surface through a surgical procedure $[12,13]$. This surgical procedure to set EEG leads could cause surgical complications such as wound infections or local brain damages which can distort the research results on sleep-wake states $[12,13]$. Also, accessory equipments of EEG can restrict free movements of animals. In order to solve these problems, remote EEG recording without electrode lines has been developed [14-16], but the remote

(c) This is an Open-Access article distributed under the terms of the Creative Commons Attribution Non-Commercial License (http://creativecommons.org/licenses/by-nc/4.0) which permits unrestricted non-commercial use, distribution, and reproduction in any medium, provided the original work is properly cited. 
EEG still has the problem of setting the EEG electrodes on the brain surface. For these reasons, it was difficult to use EEG in animal research for longitudinal study of sleepwake states.

Other than measuring sleep-wake states through EEG, behavior of animals could be used to predict sleep-wake states. Locomotor activities would be useful to predict sleep-wake states, considering that activity levels of animals drop while asleep and vice versa $[10,17,18]$. Locomotor activities have been assessed by various methods [17-20]. Wheel-running activity is widely used to measure locomotor activities in research of circadian rhythms $[21,22]$. However, wheel-running activity is suitable for measuring voluntary movements and not adaptable for measuring the wake state showing subtle motion independent of voluntary movements [18]. Recent development of experimental apparatus allowed advanced highthroughput behavioral assessments to measure locomotor activities in details. Several methods such as video based monitoring [13,18], piezo-electric sensor [23], infrared beam break detector [12], passive infrared motion sensor [24] have been tried to predict sleep-wake states using locomotor activity instead of EEG/EMG.

While each method to measure locomotor activity has its own advantages and disadvantages, the assessments using infrared detectors might be useful in several aspects $[25,26]$. Firstly, infrared detectors can monitor free movements in home cages using external detectors without surgical procedures as in EEG [24]. Additionally, the data derived from infrared detectors can be easily collected and analyzed in real time and longitudinal observations [24]. Furthermore, because it is less likely to harm the animal body, it is used for measuring movements and predicting sleep-wake states in clinical studies [27]. Thus, the findings in animal research can be conveniently expanded into clinical studies.

Several validation studies of infrared detectors on measuring locomotor activities were done in the 1980s $[28,29]$. However, there was only one validation study of infrared detectors on sleep-wake states despite of several advantages [24], and such studies using measurement devices for measuring locomotor activities have been attempted recently $[12,13,18,23,24]$. This validation study showed that the sleep states predicted by passive infrared motion sensors were highly correlated with EEG-defined sleep in four C57BL/6] mice (Pearson's $r$ > 0.95) [24]. Given that the difference of biological mechanisms between sleepwake cycles and rest-activity rhythms, proper algorithms to process the raw data of locomotion could be warranted to predict the sleep-wake states efficiently. A previous validation study considered immobilization of more than 40 seconds as sleep state $[12,24]$. This algorithm was identically used in validation studies using other devices such as video-tracking monitoring [12,13], piezo-electric sensor [23] and infrared beak breaking activity monitoring [30], and this could be reasonable when considering sleep state tends to sustain more than a certain amount of time. However, more various algorithms for data processing need to be tried to improve predictability. Also, validation studies on various strains and species, as well as various conditions are required in order to utilize this device extensively. Therefore, this study aimed to examine the prediction power of an infrared locomotion detector on sleep-wake states in ICR (Institute Cancer Research) mice that are widely used in animal studies. Lastly, we also explored the influence of the durations and methods of data processing on improvement of the prediction power using four different techniques such as data averaging, data summation, moving average, and the definition of sleep state by Pack et al. [12].

\section{METHODS}

\section{Animals}

Male ICR mice ( $\mathrm{n}=7,33-37 \mathrm{~g}, 5-6$ weeks old) were used. During the experiments, temperature and humidity were maintained $20 \pm 1{ }^{\circ} \mathrm{C}$ and $40-60 \%$ respectively. Animals were placed in cages that allowed free movements and were given ad libitum access to food and water. The cages were hexahedron shaped with bottom of $17 \times 26 \mathrm{~cm}$ and top of $18 \times 30 \mathrm{~cm}$. The mice were allowed to adapt to the new environment for 7 days. The light was turned on from 8 am to $8 \mathrm{pm}$ and turned off until next 8 am under a 12:12 light-dark cycle. This research was approved by Institutional Animal Care and Use Committee at Pusan National University Hospital (PNUH-2017-118).

\section{Measurements}

\section{Sleep-wake state}

Sleep-wake states were measured using video record- 
ings (Sony Model HDR-CX560; Sony, Tokyo, Japan). The locomotor activities of the mice in each home cage were recoded for 24 hours and their sleep-wake states were analyzed according to the determining criteria which were modified from the methods by Schwartz and Smale [11]. The criteria for determining the sleeping state were stated as follows: 1) keeping inactive states while the eyes are shut and 2) leaning the body on the wall or floor, or 3) dipping head without gross motion. On the other hand, the criteria for making decisions on the wake state were as follows: 1) moving actively, 2) eating or gnawing something, 3) grooming body, 4) standing with only two hind legs, 5) turning its head with blinking eyes with active motions and 6) exploring the environment with blinking eyes with no active motion. Vague behaviors around inactive state, such as body shivering, muscle twitching or jerky movement, were evaluated based on the degrees of features or durations that have sufficient influences on changing the sleep state. Sleep-wake states were determined as 1 (wake state) or 0 (sleep state) in the video analysis (Fig. 1).

\section{Locomotor activity}

The locomotor activities of mice were measured by using an infrared locomotion detector, Mlog system (Biobserv Inc., Bonn, Germany), which can detect infrared rays that mice emit while they are moving freely in their cages. Activities measured by this system were converted to voltage, and the values were saved as a natural number for each second according to the degrees of movement (In this study, minimum 1 to maximum 164, Fig. 1). When there was no movement, the value was recoded as zero. A larger value indicates more activity.

\section{Experiment Procedure}

Total of 7 mice were entrained into a light-dark 12:12 cycle for one week. Light was automatically controlled by Light Control System (Philips, Burlington, MA, USA). After the one-week accommodation period, sleep-wake states and locomotor activities of mice were simultaneously evaluated for 24 hours a day by using video recording and intra-red locomotion detectors under the conditions with free movement in each home cage. The light-dark condition in experiment was maintained as the same condition at the accommodation period.

\section{Data Analysis}

The receiver operating characteristic (ROC) curve analysis was used in order to evaluate the prediction power of the infrared locomotion detector on sleep-wake states. The sleep-wake states from the video analysis was considered as a true class label for the ROC curve analysis. Locomotor activities measured by Mlog system was used as a predictor. The 'perfcurve' method in Matlab is utilized to perform the ROC curve analysis. Sensitivity, specificity, positive predictive value (PPV), negative predictive value (NPV), accuracy, area under the curve (AUC) value and optimal cut-off scores were calculated by Matlab R2013b.

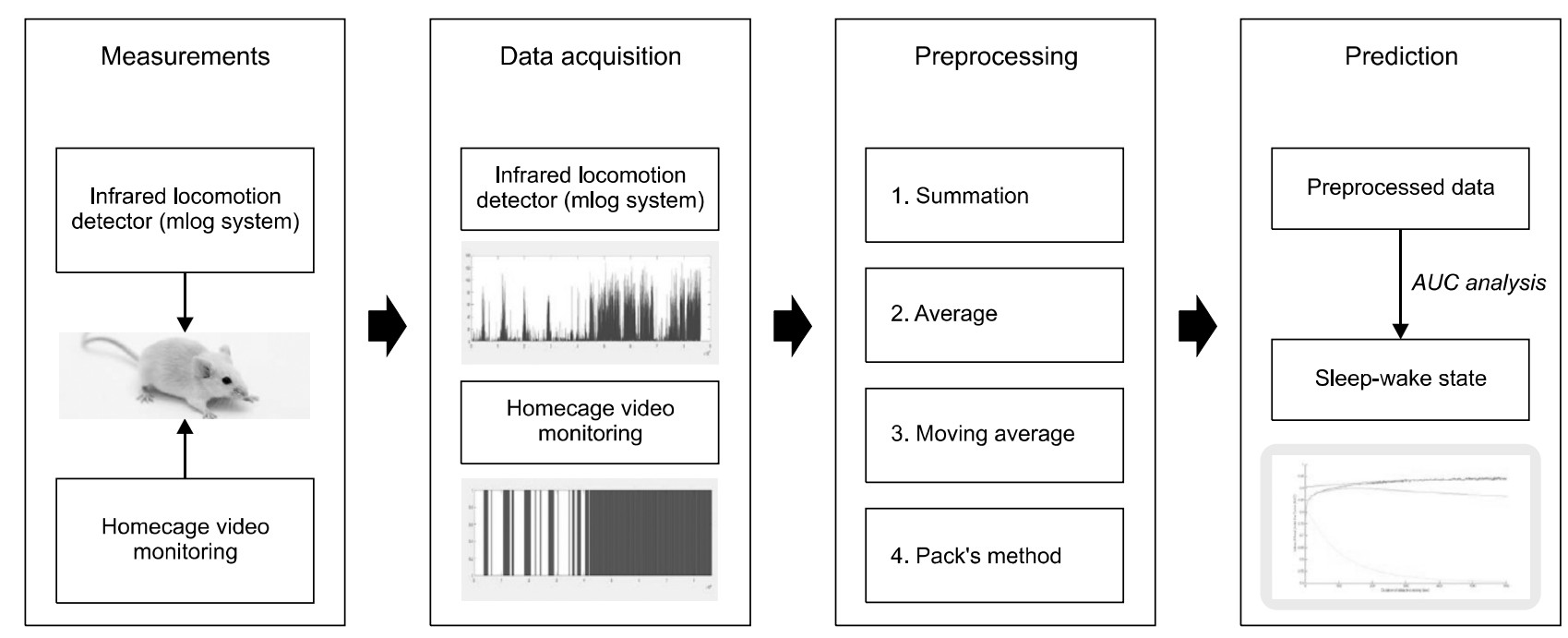

Fig. 1. The schematic process to predict sleep-wake state after preprocessing data obtained by infrared locomotion detector. AUC, area under the curve. 
For examining the influence of the duration of data processing for improvements of the prediction power, four methods of data processing, data average, data summation, moving average, and the definition of sleep state by Pack et al., were used for data processing in this study (Fig. 1). Through the process, the original data gathered per second were newly transformed into gradually increasing the duration of data processing, such as $2,3, \cdots$, 3,600 seconds. The AUC values based on newly transformed data were calculated by Matlab. As the duration of data processing increases, the changes of the AUC values were demonstrated on graphs (Fig. 1). While performing data processing of Mlog data using data averaging and summation, the data of sleep-wake states were also transformed for the new analysis, because the sleep-wake were originally evaluated per second. If the mean value of data of sleep-wake states for the duration of data processing using data averaging and summation was greater than 0.5 , the sleep-wake state was considered as wake state (1 score). Meanwhile, the moving average and permission methods used the raw data of Mlog, because the total data numbers were not changed. Additionally, the possibility of further improvements using the combination of existing ways was explored. In the results of data processing using moving average, the 120 seconds showed the maximal value of AUC. Thus, the combined method, which is the data summation after the 120 second-leading moving average, was performed additionally.

During the 10-minute period, the sensitivity, specificity, PPV, NPV, accuracy, AUC, and optimal cut-off scores were calculated at every 2-minute interval. Also, these characteristics of ROC curve analysis were compared at every 2-minute intervals in data summation by using Delong's test in the pROC package of $\mathrm{R}$ version 3.6.0. The statistical significance level was less than 0.05 in two-sided tests.

\section{RESULTS}

\section{Sleep Features in ICR Mouse Measured by Video Analysis}

Total sleep duration by the video analysis was $30,884 \pm$ $3,747.1$ seconds on average (8 hours 34 minutes 44.9 seconds) (95\% Confidence Interval [Cl], 27,418-34,329). The mean of sleeping time in the light phase was $26,523 \pm$ 1,767.9 seconds ( 7 hours 22 minutes 3.3 seconds) (95\% $\mathrm{Cl}, 24,888-28,158)$ while that of the dark phase was 43,606 $\pm 3,563.3$ seconds (1 hour 12 minutes 40.6 seconds) $(95 \% \mathrm{Cl}, 1,065.0-7,656.1)$.

\section{Initial ROC Analyses for Predicting Sleep-wake State before Data Processing}

When the cut-off score to predict sleep-wake states was set at 1 in the initial ROC curve analysis before data processing, the analysis showed acceptable sensitivity (0.615), specificity (0.936), PPV (0.945), NPV (0.575), accuracy (0.730), and AUC (0.785) (Table 1).

\section{Comparison of the AUC Values according to the Durations according to Data Processing}

As shown in Figure 2 and Table 1, in simulations using both averaging and summing data, the AUC values for prediction of sleep-wake state were calculated according to the 1-hour durations of data processing. In the initial

Table 1. Results of receiver operating curve analysis according to data summation

\begin{tabular}{|c|c|c|c|c|c|c|}
\hline Duration of data processing & 1-second & 2-minute & 4-minute & 6-minute & 8-minute & 10-minute \\
\hline Sensitivity & 0.615 & 0.813 & 0.840 & 0.880 & 0.895 & 0.889 \\
\hline Specificity & 0.936 & 0.888 & 0.912 & 0.886 & 0.866 & 0.901 \\
\hline PPV & 0.945 & 0.932 & 0.947 & 0.936 & 0.926 & 0.954 \\
\hline NPV & 0.575 & 0.715 & 0.752 & 0.796 & 0.816 & 0.778 \\
\hline Accuracy & 0.730 & 0.839 & 0.865 & 0.882 & 0.885 & 0.893 \\
\hline$A \cup C$ & 0.785 & 0.906 & 0.921 & 0.932 & 0.938 & 0.942 \\
\hline Optimal cut-off score & 1 & 76 & 161 & 185 & 243 & 313 \\
\hline \multicolumn{7}{|l|}{ DeLong's test } \\
\hline $\begin{array}{l}\text { D value ( } p \text { value) in comparison } \\
\text { of baseline data ( } 1 \text { second) }\end{array}$ & - & $\begin{array}{c}28.981 \\
(<0.001)\end{array}$ & $\begin{array}{l}25.339 \\
(<0.001)\end{array}$ & $\begin{array}{l}24.480 \\
(<0.001)\end{array}$ & $\begin{array}{l}23.098 \\
(<0.001)\end{array}$ & $\begin{array}{c}21.933 \\
(<0.001)\end{array}$ \\
\hline $\begin{array}{l}\text { D value ( } p \text { value) in comparison } \\
\text { of } 10 \text {-minute data }\end{array}$ & $\begin{array}{l}-21.933 \\
(<0.001)\end{array}$ & $\begin{array}{l}-4.389 \\
(<0.001)\end{array}$ & $\begin{array}{l}-2.359 \\
(0.018)\end{array}$ & $\begin{array}{l}-1.053 \\
(0.293)\end{array}$ & $\begin{array}{l}-0.481 \\
(0.631)\end{array}$ & - \\
\hline
\end{tabular}

PPV, positive predictive value; NPV, negative predictive value; AUC, area under the curve. 

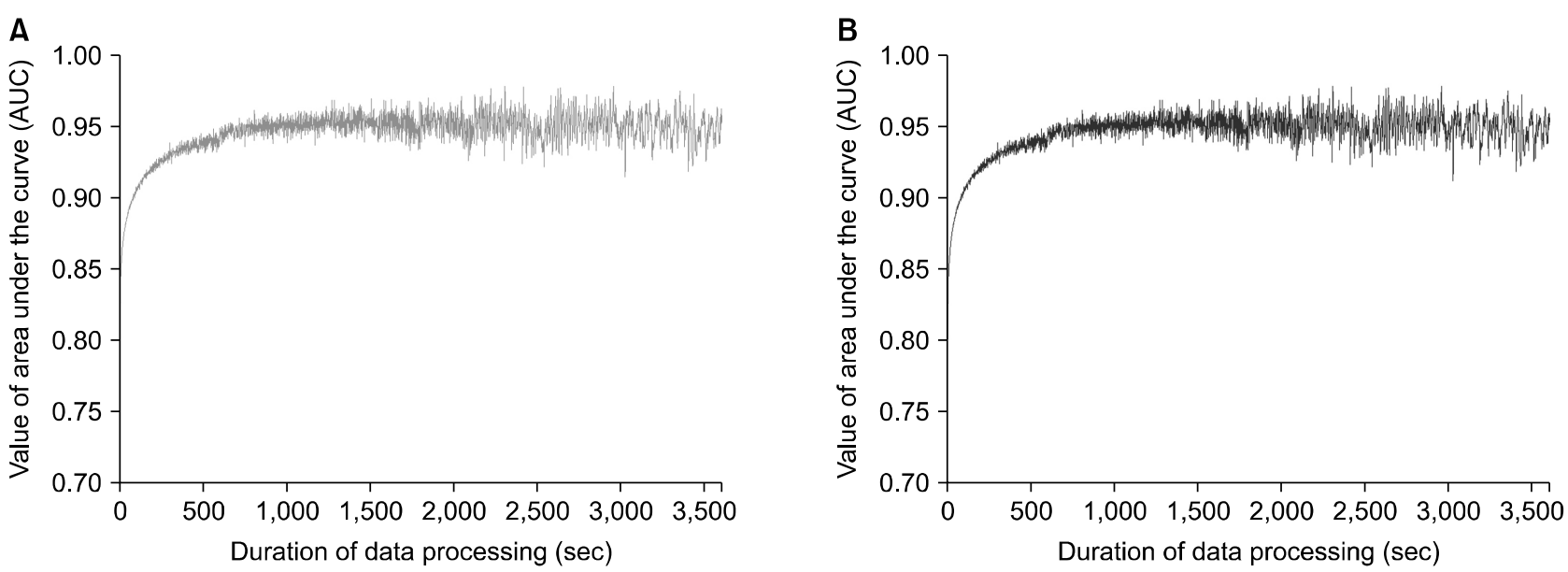

Fig. 2. Prediction of an infrared locomotor detector on the sleep-wake state according to the duration of data process such as data averaging (A) and data summation (B). (A) Red line shows the predicted values of area under the curve (AUC) after data processing using data averaging with the raw data measured by an infrared locomotor detector. As the duration of data process increases, the AUC values increase steeply within 200 seconds and hit a plateau after approximately 600 seconds. (B) Blue line indicates the predicted AUC values after data processing using data summation. The graph in data summation was identical to $(\mathrm{A})$ in data averaging.

state before the simulations, the AUC value was 0.785 . The predicted AUC values between data averaging and summation were identical. As the duration of data processing increase, the AUC values gradually increased in both methods of data processing. When using data summation, the values of AUC increased sharply before the 100 -second duration then reached to a plateau around 0.94 after the 300-second duration (Figs. 2, 3). In addition, the AUC values calculated that were within the 10-minute duration showed relatively small oscillations compared to those after the 10-minute duration.

Comparative graphs of ways of different data processing were shown in Figure 3. When using moving average, it showed similar pattern like data summation before 50 -second duration. The maximal value around $120 \mathrm{sec}$ ond was observed then the AUC value was slightly decreased around to 0.86 after the 200-second duration. The method from the definition of sleep state by Pack et al. showed an increase to 0.8 with 10 -second duration then a gradual descent to around 0.5 was observed. Combination method using the data summation after moving average with 120-second leading showed a slight increase of AUC value up to 0.94 which was almost identical to data summation after the 200-second duration.

\section{Comparison of the ROC Analyses according to Data Summation}

The ROC characteristics for predicting sleep-wake states showed an improvement after data summation for 10 minutes (Table 1 and Figs. 4, 5). The initial AUC value was 0.785 , it increased gradually to 0.942 by the 10 -minute data summation (Table 1 and Figs. 4, 5). At the 10-minute data processing, the ROC curve analysis showed improved sensitivity (0.889), specificity (0.901), PPV (0.954), NPV (0.778) and accuracy (0.893) in data summation (Table 1).

As seen in Table 1, when DeLong's tests were performed, all AUC values after the 2-minute duration of data summation were significantly higher than initial values $(p<$ 0.001). In contrast, the AUC values of 2-minute and 4-minute data summation were significantly lower than the AUC value of the 10-minute data summation (2-minute, $p<$ 0.001 ; 4-minute $p=0.018$ ). There were no significant differences between the AUC values of 6-minute and 10-minute data summation (6-minute vs. 10-minute, $p=$ 0.293; 8-minute vs. 10-minute, $p=0.631$ ).

\section{DISCUSSION}

This study investigated the prediction power of locomotor activities measured by infrared detectors on the sleep-wake states in ICR mice. We found that infrared motion detectors could predict acceptable sleep-wake states in ICR mice. Furthermore, simple data processing procedures such as data averaging and summation excellently improved the predictive power. The optimal duration of 

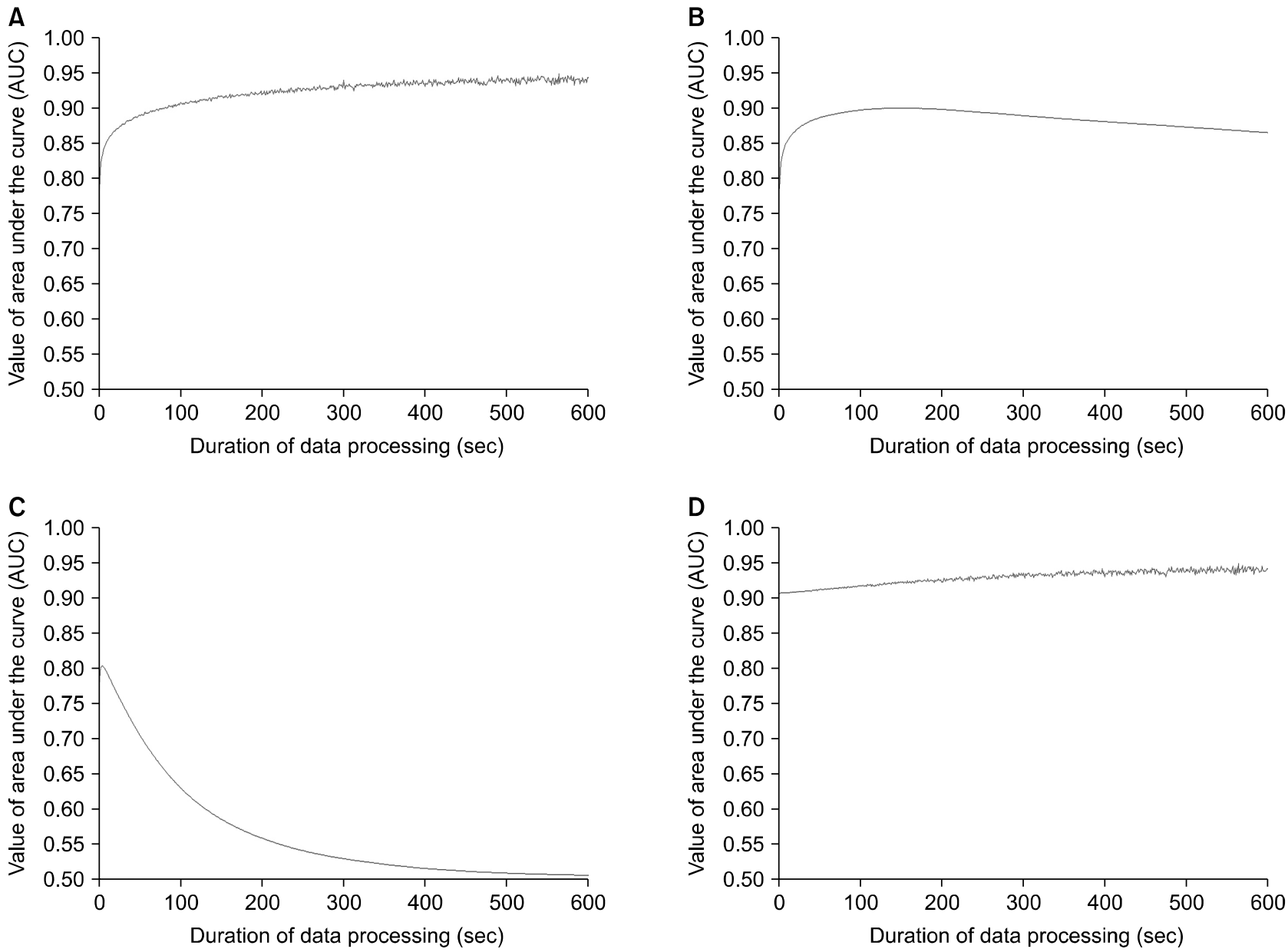

Fig. 3. Comparison of the prediction power on sleep-wake state by four methods of data processing. Data summation (A), moving average (B), tolerance (C) and data summation after moving average with a 120 second leading (D). (A) Data summation shows an initial increase, a plateau after 100 seconds and flatness around 0.91 after 300 seconds. (B) Moving average initially presents a similar pattern like data summation. There is a maximal value around 120 seconds and a slight decrease around to 0.86 after 200 seconds. (C) Permission indicates an initial increase up to 0.8 around 10 seconds and then gradual descent to around 0.5. (D) Combination method means to use the data summation after moving average with 120 second leading. Combination method shows a slight increase of the area under the curve (AUC) value up to 0.94 .

data processing might be around 8 to 10 minutes, based on the results of simulations of AUC and DeLong's test. This device might be one of the useful measurements of sleep-wake states in ICR mice. In this study, the total sleep time was estimated to be approximately 8.5 hours despite the differences according to the preprocessing methods. One comprehensive review reported that total sleep times ranged from 6.2 to 14.9 hours in mice and from 13.2 to 15.3 hours in rats [31]. Total sleep time could be influenced by various factors such as light conditions, genetic strains and ages [31-33]. If there was a longer light period, nocturnal animals slept more [31]. In addition, it is possible to have different genetic strains between short and long sleepers [33]. Furthermore, mice and rats during peri-adolescent period show shorter sleep time compared to matured rodents $[32,34]$. In this study, short sleeping of ICR mice at the peri-adolescent period (5-6 weeks old) was similar to previous findings [31-33].

The infrared motion detector (Mlog system; Biobserv Inc.) was used in this study to measure locomotor activities for each second. Even though it is a high-throughput device, the AUC value using the raw data from Mlog system before data processing might be just acceptable [35] After data processing, the AUC for prediction of sleepwake states in ICR mice maximally increased up to 0.942. The results of this study were similar to those of a previous validation study with $\mathrm{C} 57 \mathrm{BL} / 6 \mathrm{~J}$ mice [24]. Also, these results were in lines with those when other devices to meas- 


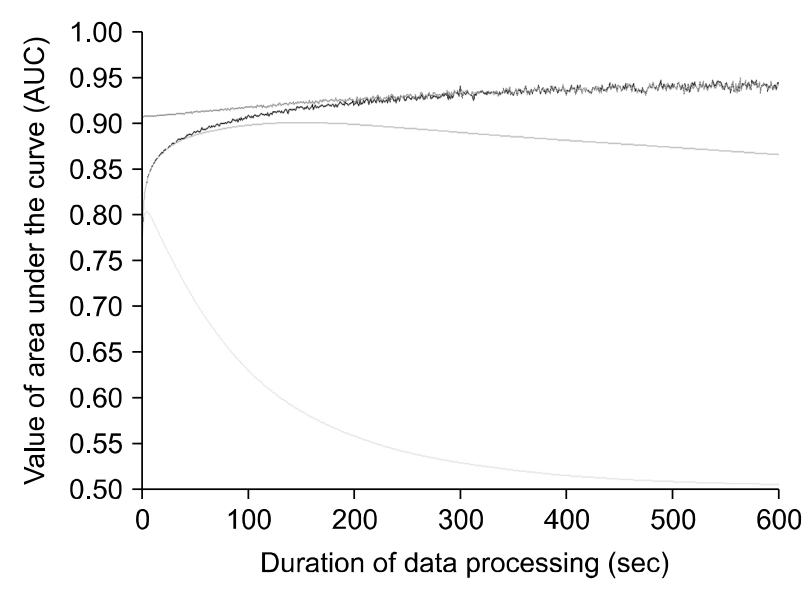

Fig. 4. Comparison of the prediction power on sleep-wake state by four methods of data processing. Data summation (blue line), moving average (margenta line), tolerance (cyan line) and data summation after a 120-second leading moving average (red line). Data summation (blue line) shows an initial increase before 100-second, a plateau around 0.94 after 300 seconds. Moving average (margenta line) initially displays a similar pattern like data summation and the maximal area under the curve (AUC) value is observed around the 120 seconds. Then, the AUC value slightly decreased to around 0.86 after the 200 seconds. Permission way (cyan line) presents the initial increase up to 0.8 around in 10 seconds and then gradual descent to around 0.5 is observed. Combination way (red line) using the data summation after the 120-second leading moving average shows a slight increase of the AUC value up to 0.94 almost identical to data summation after the 200 second durations.

ure locomotor activities were used. In a study of video analysis with $\mathrm{C} 57 \mathrm{BL} / 6$ mice, it showed high correlation (correlation coefficient 0.94) and excellent sensitivity (95\%) between EEG/EMG and digital video analysis [13]. Another study that monitored infrared beam breaks in $\mathrm{C} 57 \mathrm{BL} / 6 \mathrm{~J}$ mice reported a concordance rate of $92 \%$ between EEG and infrared beam measurements [12]. In addition, one study using a single Polyvinylidine Difluoride sensor on the cage floor to detect motion pressure showed a high classification rate (94\%) for predicting sleep-wake states in C57BL/6J mice [36]. Therefore, locomotor activities measured by infrared detectors could be helpful to predict the sleep-wake states like other devices.

In this study, the four methods of data processing were simulated. The results of simulation using data averaging were identical to those of data summation, though we simply expected that these two ways could make a different prediction. However, due to the differences in calculation processes, the interpretability of optimal cut-off value may differ. That is, in case of data summation, the cut-off value would be integers, meanwhile the cut-off

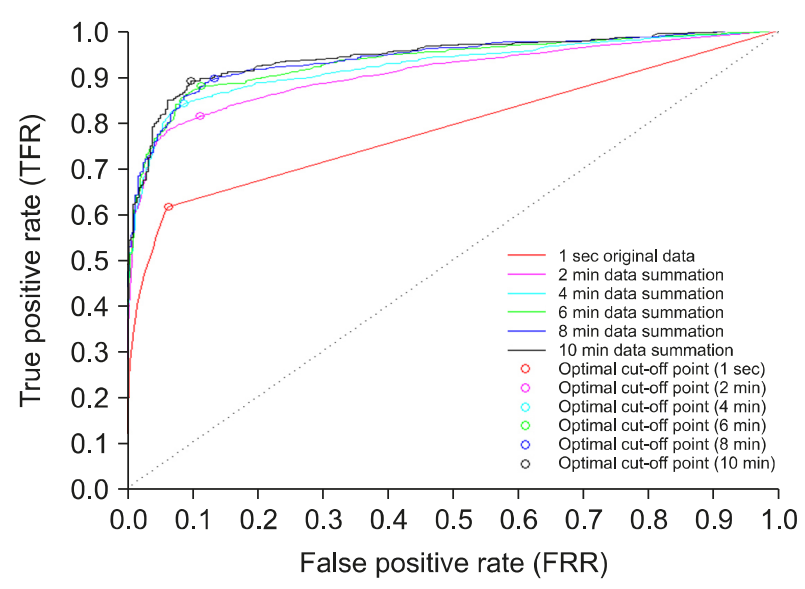

Fig. 5. Comparison of the receive operating characteristic (ROC) curve according to the durations of data processing. The ROC curves and optimal cut-off points using data summation according to the durations of data processing (original 1 second, 2 minutes, 4 minutes, 6 minutes, 8 minutes, 10 minutes) are described. As the durations of data summation increases, the area under the curve values are increased as well. The optimal cut-off points according to the durations of data summation are represented as circles.

value of the data average must be real numbers that are in decimals. Thus, the cut-off value of data summation would be easy to interpret the implication. The moving averaging way is widely used in analysis of time series data [37]. In this study, this way also showed an acceptable AUC value approximately 0.9 on the prediction of sleep-wake state around 120-second duration. When observing the results of simulation, the optimal range might be wider from 60-second to 300-second duration. Interestingly, the AUC value when using moving average were almost maintained without huge variations within 10-miniutue duration. Thus, this way might be an alternative method in order to stably analyze the data after removing unnecessary noise.

Meanwhile, the way to consider the duration of immobility showed relatively a low prediction power except the initial 10-second duration. In previous studies, they used an algorithm that considered immobility longer than 40 seconds as sleep state $[12,13,23]$. This algorithm is likely to adapt the characteristics of sleep continuity. Conceptually, this algorithm is similar to moving average. However, when the value shows immobility less than the criteria of duration, the data cannot be considered as sleep state despite the immobility like sleep. Thus, if the rest-activity cycles is destabilized without sustaining sleep-wake cycles, the predictive power of this method 
could decrease [38]. The previous studies indicated the difference between younger and older aged animals $[12,23]$. When using this way, it needs to be considered that the optimal duration to make decision on the criteria of immobility might be different under the physical conditions or in strains or species.

We also simulated the combination method to use data summation after 120-second leading moving average that showed the maximal predictive power. Interestingly, the results of the combination method became similar to those of the simple data summation. Data summation is a simple algorithm but can reflect total trends during a specific interval. Since the moving average was conceptually similar to simple data summation, it is possible that combining these methods may not show improved results. After all, the reason that data processing can improve the prediction power of an infrared locomotion detector on sleep-wake states would be based on the basic concept. Total trends during specific intervals would be better for making predictions on sleep-wake states than immobility at a specific time point. In case of immobility due to sleep, because a sleep state tends to last more than several minutes, it is likely that the animals did not move for a few minutes. In contrast, without any motion in the wake state, even during periods of low activity, it is likely to move a little within minutes. It is possible that a simple data processing algorithm has an important role on the biological link between locomotor activities and sleep-wake cycles. Therefore, this method might have a crucial role in making locomotor activity predictions effectively in sleep-wake states.

There are some limitations in this study. Firstly, the number of animal used in this study was small to generalize these findings, although the sample size was similar or larger than previous studies $[12,13,24,36]$. Secondly, we did not use EEG to evaluate sleep-wake status. Studies investigating sleep-wake cycles normally use both EEG analysis and video analysis to increase accuracy $[25,26]$, but our research evaluated sleep-wake cycles only by video analysis. However, considering the high agreement rate between EEG and video analysis in previous research, it is assumable that video analysis alone could accurately evaluate sleep-wake cycles [12]. Furthermore, because free movements are essential based on the purpose of this study, we put more weight on free locomotion than precision. Thirdly, this study did not compare the effec- tiveness among other complex algorithms of data processing like machine learning. Also, we did not adapt algorithms to predict detailed sleep parameters, such as sleep onset, sleep offset and sleep fragmentation. In the future research, in order to use infrared locomotor detectors efficiently, these various algorithms to improve prediction powers are needed to be employed. Fourthly, this study only used ICR mouse in peri-adolescent period. These results were limited to peri-adolescent ICR mice. However, given that various sleep patterns in peri-adolescent mice compared to matured adult mice [32], these results might be potentially extended into adult ICR mice that show relatively stable sleep-wake patterns. The prediction powers of locomotor activities measured by an infrared motion detector on sleep-wake states in various ages, different strains of mouse or other species such as rat are necessary. Fifthly, this study was performed only in healthy animals. In order to confirm these findings, the future studies are needed in animal models such as depression, hyperlocomotion or insomnia.

Despite these limitations, this study showed significance that locomotor activities measured by an infrared motion detector in home cages can predict sleep-wake states in ICR mice. Furthermore, simple data summation satisfactorily improved the predictive power of locomotor activities on sleep-wake states. Therefore, infrared locomotion detectors and data processing methods might be useful in animal research on sleep-wake states or circadian rhythms. Given that similarity of actigraphy in human research, the extension of these findings into human research could be meaningful for appropriate preprocessing of behavioral data that collected longitudinally. Furthermore, translational research on sleep-wake circadian rhythm using locomotion or gross movement might be facilitated.

An infrared locomotion detector predicted effectively sleep-wake states in ICR mice. After the optimal data processing, the prediction power was improved, and this infrared locomotion detector has advantages that can measure free movements of animals in their home cages without any restrictions longitudinally. Thus, this device could be one of useful measurement tools in animal research with longitudinal observations on sleep-wake cycles or circadian sleep-wake rhythms. 


\section{Acknowledgments}

This work was supported by the National Research Foundation of Korea (NRF) grant funded by the Korea government (MSIT) (No. NRF-2017R1C1B5074937).

\section{- Conflicts of Interest}

No potential conflict of interest relevant to this article was reported.

\section{Author Contributions}

Conceptualization: Min Soo Jung, Eunsoo Moon, Chi Eun Oh, and Jung Hyun Lee. Data acquisition: Jeonghyun Park, Min Soo Jung, and Hyun Ju Lim. Formal analysis: Jeonghyun Park and Eunsoo Moon. Funding: Eunsoo Moon. Supervision: Eunsoo Moon. Writing - original draft: Jeonghyun Park and Eunsoo Moon. Writing-review \& editing: Min Soo Jung, Hyun Ju Lim, Chi Eun Oh, Jung Hyun Lee, and Eunsoo Moon.

\section{ORCID}

Jeonghyun Park https://orcid.org/0000-0003-1050-212X Min Soo Jung https://orcid.org/0000-0003-4208-0267 Eunsoo Moon https://orcid.org/0000-0002-8863-3413 Hyun Ju Lim https://orcid.org/0000-0002-8962-8173 Chi Eun Oh https://orcid.org/0000-0002-0439-8170 Jung Hyun Lee https://orcid.org/0000-0002-0496-9826

\section{REFERENCES}

1. Nestler EJ, Hyman SE. Animal models of neuropsychiatric disorders. Nat Neurosci 2010;13:1161-1169.

2. Zhang JC, Toyohara J, Wu J, Ishiwata K, Hashimoto K. In vivo evaluation of ${ }^{11} \mathrm{C}$-labeled three radioligands for glycine transporter 1 in the mouse brain. Clin Psychopharmacol Neurosci 2012;10:34-43.

3. Shirai Y, Fujita Y, Hashimoto K. Effects of the antioxidant sulforaphane on hyperlocomotion and prepulse inhibition deficits in mice after phencyclidine administration. Clin Psychopharmacol Neurosci 2012;10:94-98.

4. Song JC, Seo MK, Park SW, Lee JG, Kim YH. Differential effects of olanzapine and haloperidol on MK-801-induced memory impairment in mice. Clin Psychopharmacol Neurosci 2016;14:279-285.

5. Choi HJ, Im SJ, Park HR, Park S, Kim CE, Ryu S. Long-term effects of aripiprazole treatment during adolescence on cognitive function and dopamine D2 receptor expression in neurodevelopmentally normal rats. Clin Psychopharmacol Neurosci 2019;17:400-408.
6. Einberger C, Puckett A, Ricci L, Melloni R Jr. Contemporary pharmacotherapeutics and the management of aggressive behavior in an adolescent animal model of maladaptive aggression. Clin Psychopharmacol Neurosci 2020;18:188-202.

7. Paterson LM, Nutt DJ, Wilson SJ. Sleep and its disorders in translational medicine. I Psychopharmacol 2011;25:1226-1234.

8. Campbell IG. EEG recording and analysis for sleep research. Curr Protoc Neurosci 2009; Chapter 10:Unit10.2.

9. Balzamo E, Van Beers P, Lagarde D. Scoring of sleep and wakefulness by behavioral analysis from video recordings in rhesus monkeys: comparison with conventional EEG analysis. Electroencephalogr Clin Neurophysiol 1998;106:206-212.

10. Nicolau MC, Akaârir M, Gamundí A, González J, Rial RV. Why we sleep: the evolutionary pathway to the mammalian sleep. Prog Neurobiol 2000;62:379-406.

11. Schwartz MD, Smale L. Individual differences in rhythms of behavioral sleep and its neural substrates in Nile grass rats. J Biol Rhythms 2005;20:526-537.

12. Pack Al, Galante RJ, Maislin G, Cater J, Metaxas D, Lu S, et al. Novel method for high-throughput phenotyping of sleep in mice. Physiol Genomics 2007;28:232-238.

13. Fisher SP, Godinho SI, Pothecary CA, Hankins MW, Foster RG, Peirson SN. Rapid assessment of sleep-wake behavior in mice. J Biol Rhythms 2012;27:48-58.

14. Güler NF, Ubeyli ED. Theory and applications of biotelemetry. J Med Syst 2002;26:159-178.

15. Kramer K, Kinter LB. Evaluation and applications of radiotelemetry in small laboratory animals. Physiol Genomics 2003;13:197-205.

16. Weiergräber M, Henry M, Hescheler J, Smyth N, Schneider T. Electrocorticographic and deep intracerebral EEG recording in mice using a telemetry system. Brain Res Brain Res Protoc 2005; 14:154-164.

17. Storch C, Höhne A, Holsboer F, Ohl F. Activity patterns as a correlate for sleep-wake behaviour in mice. J Neurosci Methods 2004;133:173-179.

18. Bains RS, Wells S, Sillito RR, Armstrong JD, Cater HL, Banks $\mathrm{G}$, et al. Assessing mouse behaviour throughout the light/dark cycle using automated in-cage analysis tools. I Neurosci Methods 2018;300:37-47.

19. Pasquali V, Scannapieco E, Renzi P. Validation of a microwave radar system for the monitoring of locomotor activity in mice. J Circadian Rhythms 2006;4:7.

20. Yaghouby F, Donohue KD, O'Hara BF, Sunderam S. Noninvasive dissection of mouse sleep using a piezoelectric motion sensor. J Neurosci Methods 2016;259:90-100.

21. Banks GT, Nolan PM. Assessment of circadian and light-entrainable parameters in mice using wheel-running activity. Curr Protoc Mouse Biol 2011;1:369-381.

22. Eckel-Mahan K, Sassone-Corsi P. Phenotyping circadian rhythms in mice. Curr Protoc Mouse Biol 2015;5:271-281.

23. Flores AE, Flores JE, Deshpande H, Picazo JA, Xie XS, Franken $\mathrm{P}$, et al. Pattern recognition of sleep in rodents using piezo- 
electric signals generated by gross body movements. IEEE Trans Biomed Eng 2007;54:225-233.

24. Brown LA, Hasan S, Foster RG, Peirson SN. COMPASS: continuous open mouse phenotyping of activity and sleep status. Wellcome Open Res 2016;1:2.

25. Brambilla D, Barajon I, Bianchi S, Opp MR, Imeri L. Interleukin-1 inhibits putative cholinergic neurons in vitro and REM sleep when microinjected into the rat laterodorsal tegmental nucleus. Sleep 2010;33:919-929.

26. Morrow JD, Vikraman S, Imeri L, Opp MR. Effects of serotonergic activation by 5-hydroxytryptophan on sleep and body temperature of C57BL/6J and interleukin-6-deficient mice are dose and time related. Sleep 2008;31:21-33.

27. Nakano T, Koyama E, Nakamura T, Ito T, Tamura K, Yaginuma M. Use of an infrared sensor system to take long-term bedside measurements of rest-activity patterns in the elderly with dementia. Psychiatry Clin Neurosci 2002;56:287-288.

28. Büttner D. The use of passive infrared detectors to measure locomotor activity of laboratory animals. Z Versuchstierkd 1988;31:137-141.

29. Tamborini P, Sigg H, Zbinden G. Quantitative analysis of rat activity in the home cage by infrared monitoring. Application to the acute toxicity testing of acetanilide and phenylmercuric acetate. Arch Toxicol 1989;63:85-96.

30. Naidoo N, Ferber M, Master M, Zhu Y, Pack Al. Aging impairs the unfolded protein response to sleep deprivation and leads to proapoptotic signaling. J Neurosci 2008;28:6539-6548.

31. Campbell SS, Tobler I. Animal sleep: a review of sleep duration across phylogeny. Neurosci Biobehav Rev 1984;8:269-300.

32. Nelson AB, Faraguna U, Zoltan JT, Tononi G, Cirelli C. Sleep patterns and homeostatic mechanisms in adolescent mice. Brain Sci 2013;3:318-343.

33. Dowell R, Odell A, Richmond P, Malmer D, Halper-Stromberg E, Bennett B, et al. Genome characterization of the selected long- and short-sleep mouse lines. Mamm Genome 2016;27: 574-586.

34. Gradwohl G, Berdugo-Boura N, Segev Y, Tarasiuk A. Sleep/wake dynamics changes during maturation in rats. PLoS One 2015;10:e0125509.

35. Mandrekar JN. Receiver operating characteristic curve in diagnostic test assessment. J Thorac Oncol 2010;5:1315-1316.

36. Donohue KD, Medonza DC, Crane ER, O'Hara BF. Assessment of a non-invasive high-throughput classifier for behaviours associated with sleep and wake in mice. Biomed Eng Online 2008;7:14.

37. Carbone A, Castelli G, Stanley HE. Analysis of clusters formed by the moving average of a long-range correlated time series. Phys Rev E Stat Nonlin Soft Matter Phys 2004;69(2 Pt 2): 026105.

38. Wimmer ME, Rising J, Galante RJ, Wyner A, Pack Al, Abel T. Aging in mice reduces the ability to sustain sleep/wake states. PLoS One 2013;8:e81880. 